\title{
Hierarchically Structured Mixed Oxide Electrodes for Oxygen Evolution Reaction: A Multimodal Electron Microscopy Study
}

\author{
Jingshan Du ${ }^{1}$, Qian Rong ${ }^{1}$, Xinqi Chen ${ }^{1}$, Qingju Liu ${ }^{2}$ and Vinayak Dravid ${ }^{1}$ \\ ${ }^{1}$ Northwestern University, Evanston, Illinois, United States, ${ }^{2}$ Yunnan University, Kunming, Yunnan, \\ China (People's Republic)
}

The lack of highly active, durable, and reproducible electrocatalysts for oxygen evolution reaction (OER) has been a bottleneck towards cost-effective and practical water splitting reactions [1]. In recent years, earth-abundant transition metal oxide nanostructures, for example $\mathrm{NiO}$ nanosheets (NSs), have been reported as OER electrocatalysts [2,3]. However, the catalytic performance and durability of these materials have not met commercial requirements, therefore they are not yet able to replace the rare and expensive Ir- and Ru-based electrocatalysts [4]. In this work, we describe a method for synthesizing nanoporous or nanobranched hierarchical structures consisting of up to four metal elements and three levels of structural hierarchy on the scales from few-nm to sub- $\mu \mathrm{m}$ and then sub-mm; such complexity required innovative use of multimodal microscopy and characterization techniques, including correlated electron diffraction and high-resolution transmission electron microscopy (TEM) imaging, to identify the phase structures and their geometric distribution.

Hierarchical NSs were synthesized through NS growth on ITO-coated glass plates, reacting the NSs with modifier solutions based on metal salts in acetone, and subsequent calcination (Fig. 1A). When cobalt chloride was used in the modifier solution, CoNi-O porous NSs (pNSs) were formed, and when cobalt nitrate was used, CoNi-O branched NSs (bNSs) were formed as confirmed by scanning electron microscopy (SEM). Ensemble-scale characterization shows that these materials are mainly in a rock-salt $\mathrm{NiO}$ crystal structure (Fig. 1B) and the specific surface area was increased compared to the unmodified NiO NSs (Fig. 1C). In order to further reveal the nanoscale phase structures in these materials, we prepared powders of these NS samples and performed TEM analysis. TEM imaging reveals a large portion of nanoscale pores on the pNS sample. We further acquired selected area electron diffraction (SAED) on the pNSs and radially integrated the pattern into a one-dimensional profile, which was then compared to simulated polycrystal electron diffraction patterns (Fig. 1D, middle panel); this confirms that in addition to rock-salt $\mathrm{NiO}$, a spinel $\mathrm{Co}_{3} \mathrm{O}_{4}$ phase also exists. The spatial distribution of these domains were revealed by filtering the high-resolution TEM (HRTEM) images using dominant reflections from $\mathrm{Co}_{3} \mathrm{O}_{4}$ in the reciprocal space (Fig. 1D, right panel). The phase structure of the bNSs was similarly determined. Interestingly, branch-like $\mathrm{Co}_{3} \mathrm{O}_{4}$ domains formed on continuous $\mathrm{NiO}$ thin nanosheets, and no nanopore formation was observed (Fig. 1E). The preparation of pNSs and bNSs using metal salts is generally applicable and can be used to incorporate other types of metals in the system. For instance, the use of $\mathrm{Mn}\left(\mathrm{NO}_{3}\right)_{2}$ in the modifier solution leads to the formation of MnNi-O bNSs comprised of highly defected bixbyite $\mathrm{Mn}_{2} \mathrm{O}_{3}$ nanoparticle domains supported on NiO NSs (Fig. 1F). We discovered that the choice of chlorides or nitrates for the metal salts results in different metal cluster stability in acetone and therefore controls the direction of nanomodification (Fig. 1G). Specifically, with metal chlorides, stable metal clusters are formed in acetone, which facilitates $\mathrm{Ni}$ dissolution from the nanosheets into the solution, resulting in the porous structures. With metal nitrates, however, metal cluster growth is unstable. Therefore, Ni dissolution from the nanosheets is suppressed, while the hydrolysis and decomposition of metal nitrates result in the deposition of the branched features. 
These materials were further synthesized on $\mathrm{Ni}$ foams for enhanced substrate conductivity and porosity (Fig. 2A and B). By subsequently modifying the $\mathrm{NSs}$ in $\mathrm{FeCl}_{3}, \mathrm{Co}\left(\mathrm{NO}_{3}\right)_{2}$, and/or $\mathrm{Mn}\left(\mathrm{NO}_{3}\right)_{2}$, two multimetal oxide NS materials were identified to have record-low Tafel slopes ever reported (21.5 and 22.3 $\mathrm{mV} \cdot \mathrm{dec}^{-1}$ with $i R$ correction). Importantly, these materials possess high batch-to-batch reproducibility (Fig. 2E) and excellent durability during week-long operation tests (Fig. 2F). Taken together, this design strategy for constructing hierarchical mixed oxide electrodes paves avenues towards practically robust, low-cost, and high-performance water splitting devices. Importantly, this work highlights how multimodal electron microscopy techniques can be used correlatively to elucidate the hierarchical structures in complex, multicomponent nanomaterial systems [5].
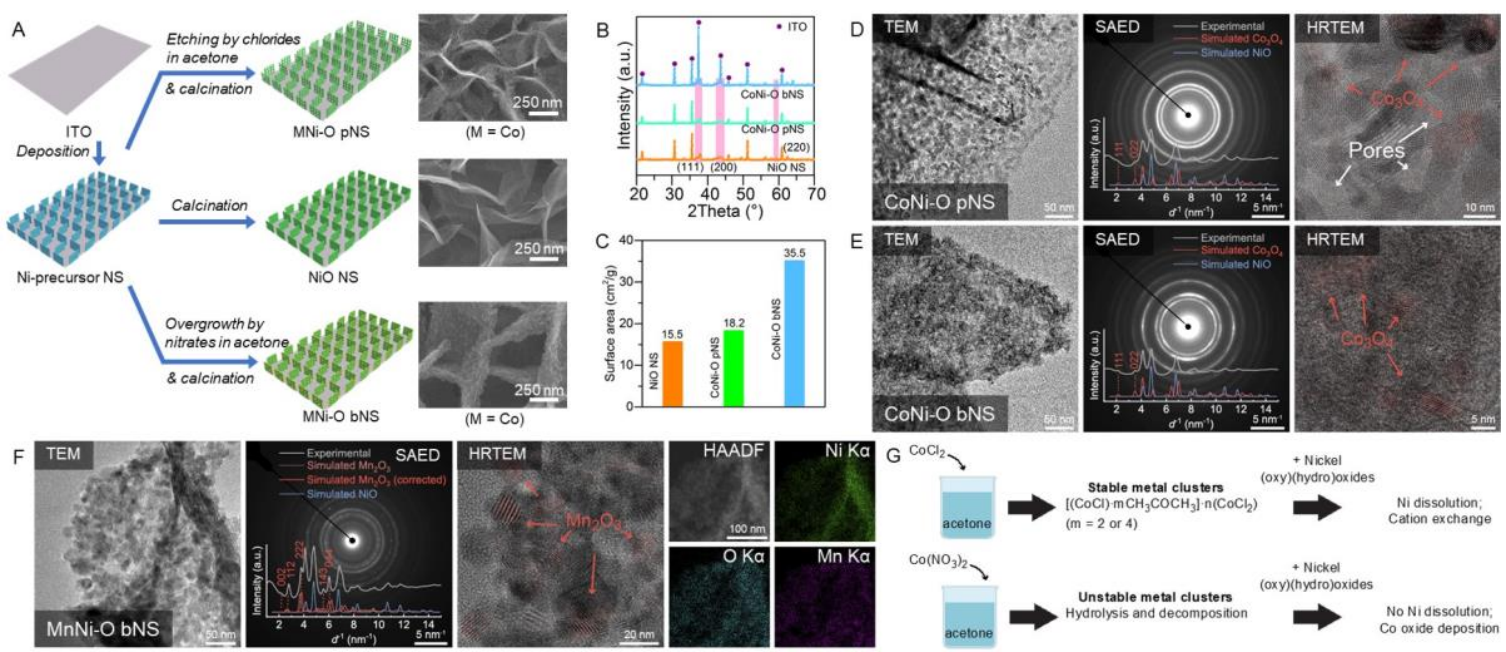

Figure 1. Porous and branched nanosheets (pNSs and bNSs) synthesized by bidirectional nanomodification with metal salts in acetone. A) Schematic illustration of the preparation procedures for MNi-O NSs (M: Fe, Co and Mn) and secondary electron (SE) images of CoNi-O pNSs, NiO NSs, and CoNi-O bNSs grown on ITO. (B) XRD patterns. (C) Specific surface area derived from N2 adsorptiondesorption experiments. (D-F) Transmission electron microscopy (TEM) characterization of secondary nanostructures formed on modified NS samples: CoNi-O pNS (D), CoNi-O bNS (E), and MnNi-O bNS (F). Experimental selected area electron diffraction (SAED) patterns are radially integrated, background subtracted, and compared with simulations based on pure $\mathrm{NiO}$ (Fm-3m), Co3O4 (Fd-3m), and Mn2O3 (Ia-3) phases. The simulated $\mathrm{Mn} 2 \mathrm{O} 3$ lattice is corrected by lattice compression to match the experimental results, presumably reflecting the defects and distortion in Mn2O3. High-resolution TEM (HRTEM) images are filtered in the reciprocal space to highlight Co or Mn oxide lattices based on the distinct crystal planes (dashed lines in SAED radial patterns), colored in red, and then stacked with the original image. Correlative high-angle annular dark field (HAADF) imaging and energy-dispersive X-ray spectroscopy (EDS) elemental mapping results further show the nanoscale structural inhomogeneity in the Mnincorporated sample. $(G)$ Proposed mechanism for the nanomodification process. 
A

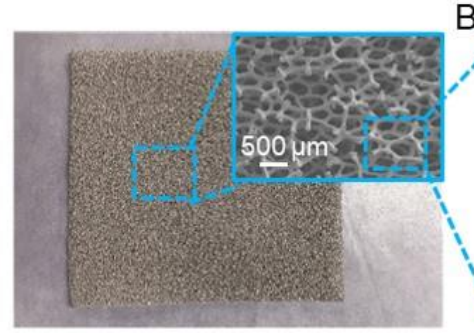

$\mathrm{D}$

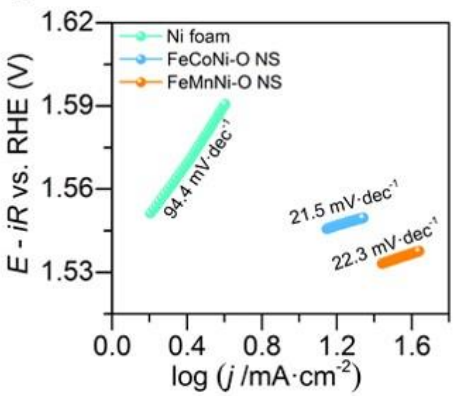

B

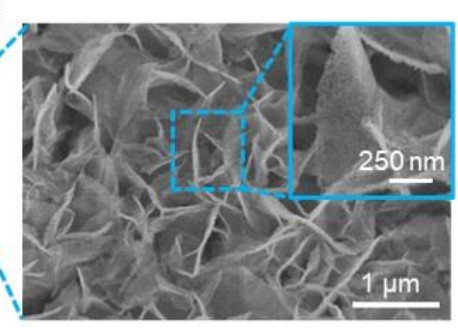

$\mathrm{E}$

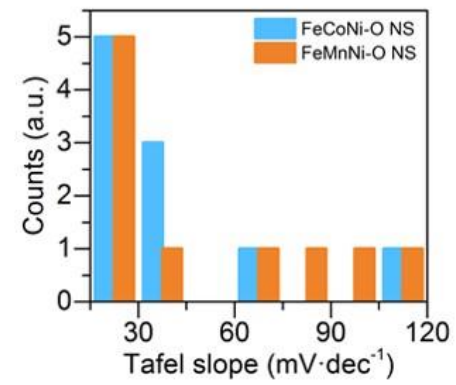

C

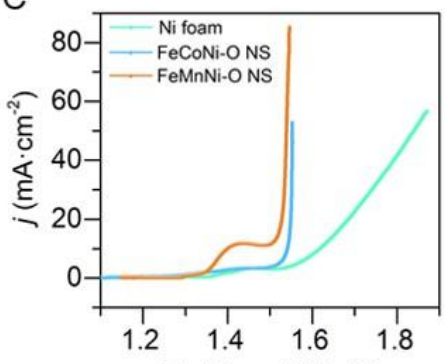

$\mathrm{F}$

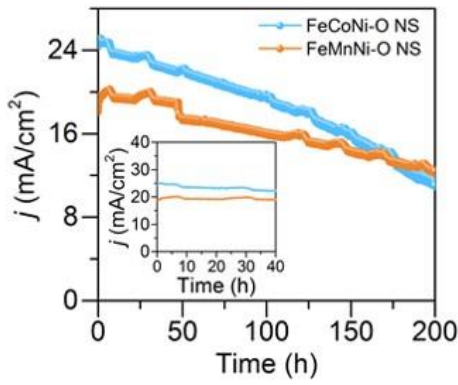

Figure 2. Oxygen evolution performance of hierarchical structured electrodes. (A) Photo of a Ni foam. Inset: low-magnitude SE image showing the Ni foam after sample deposition. (B) SE image of the hierarchical FeCoNi-O NSs on Ni foam. Inset: Enlarged SE image of an individual nanosheet. (C, D) Linear sweep voltammetry (C) and Tafel polarization curves (D) of the samples supported on Ni foam. (E) Repeatability of the best-performing samples shown by the distribution of Tafel slope by samples synthesized from different batches. (F) Long-term performing stability of FeCoNi-O NSs and FeMnNi-O NSs on Ni foam shown by current density at a constant voltage (1.55 and $1.53 \mathrm{~V}$ vs. RHE, respectively) in $0.1 \mathrm{M} \mathrm{KOH}$ electrolyte, Inset: Current density within the first $40 \mathrm{~h}$ of operation time.

\section{References}

[1] Y. Liu, Q. Li, R. Si, G. D. Li, W. Li, D. P. Liu, D. Wang, L. Sun, Y. Zhang, X. Zou, Adv. Mater. 2017, $29,1606200$.

[2] H. Kakizaki, H. Ooka, T. Hayashi, A. Yamaguchi, N. B. Mercier, K. Hashimoto, R. Nakamura, Adv. Funct. Mater. 2018, 28, 1706319.

[3] M. Gong, H. J. Dai, Nano. Res. 2015, 8, 23-39.

[4] J. S. Kim, B. Kim, H. Kim, K. Kang, Adv. Energy Mater. 2018, 8, 1702774.

[5] The authors acknowledge support from the NUANCE Center and SHyNE Resource (NSF ECCS1542205). Q.R. acknowledges a China Scholarship Council (CSC) Scholarship. 